\title{
Expression of adiponectin receptors 1 and 2 and the leptin receptor in human adrenal tumors
}

\author{
Anna Babińska ${ }^{1}$, Rafał Pęksa², Piotr Wiśniewski ${ }^{1}$, Krzysztof Sworczak $^{1}$
}

'Department of Endocrinology and Internal Medicine, Medical University of Gdansk, Gdansk, Poland

${ }^{2}$ Department of Pathology, Medical University of Gdansk, Gdansk, Poland

Submitted: 30 January 2017

Accepted: 7 May 2017

Arch Med Sci 2019; 15 (5): 1254-1260

DOI: https://doi.org/10.5114/aoms.2018.76142

Copyright @ 2018 Termedia @ Banach

\section{Abstract}

Introduction: The role of adipokines in neoplasms not related to obesity is unclear. The presence of adiponectin receptors 1 and 2 (AdipoR 1 and AdipoR2) as well as the leptin receptor (Ob-R) has been recognized in human adrenal tumors. The authors of the present study were the first to compare the expression of these receptors in histopathologically distinct adrenal tumors.

Material and methods: The study encompassed tissue specimens of 128 patients with adrenal tumors ( 28 adrenal cortical adenomas (CA), 35 cortical nodular hyperplasia tumors (CNH), 20 cortical carcinomas (CC), 40 pheochromocytomas (PHEO), 5 malignant pheochromocytomas (PHEOM)) operated on at a single clinical center. The expression of the adiponectin receptors AdipoR1 and AdipoR2 as well as the leptin receptor Ob-R was assessed by immunohistochemistry. The results were correlated with body mass index (BMI) and gender of the patients.

Results: AdipoR1 expression was significantly higher in cortical cancers ( $p<$ $0.001)$ and pheochromocytomas $(p<0.001)$ as compared to benign cortical tumors. AdipoR2 expression was significantly higher in cortical carcinomas as compared to cortical adenomas and hyperplasia tumors $(p=0.01)$, and also significantly higher in pheochromocytomas in comparison to adrenocortical cancers $(p=0.004)$. Leptin receptor expression was absent or minimal in half of nodular hyperplasia tumors and adrenal cortex adenomas. This receptor's expression was significantly higher in adrenocortical cancers ( $p=0.038$ ). In pheochromocytomas this receptor was expressed more abundantly than in adrenocortical cancers $(p=0.004)$.

Conclusions: These novel findings suggest that adiponectin and leptin receptors could play a regulatory role in human adrenal neoplasms.

Key words: AdipoR1 and AdipoR2 receptors, leptin Ob-R receptor, adrenal tumors.

\section{Introduction}

The adipose tissue is considered the largest endocrine "organ" of the human body. The higher the body mass is, the greater is the risk of type 2 diabetes mellitus and cardiovascular diseases, and also certain neoplasms.

Over 50 hormones and cytokines are synthesized and secreted by the adipose tissue [1]. In numerous reports data concerning a relationship between obesity and many cancers have been published.

\author{
Corresponding author: \\ Anna Babińska MD \\ Department \\ of Endocrinology \\ and Internal Medicine \\ Medical University \\ of Gdansk \\ 7 Dębinki St \\ 80-288 Gdansk, Poland \\ Phone: +48 583492840 \\ Fax: +48 583492841 \\ E-mail:a.mail@wp.pl
}


In the obese carcinogenesis is mediated through insulin resistance, hyperinsulinemia, and increased levels of insulin-like growth factor 1 (IGF-1) [1, 2]. In vitro studies unambiguously indicate a proliferative effect of insulin, which also inhibits apoptosis and stimulates the synthesis of IGF-1, a pro-neoplastic hormone [2].

Relationships between adipokines and carcinogenesis are also sought in neoplasms not associated with obesity [2, 3]. Therefore, the authors analyzed adipokine receptors in the human adrenal tumors, expression of which has not been widely investigated so far.

Although adiponectin is a key mediator in the development and progression of various cancers, the underlying pathomechanisms connected with neoplasia are still poorly understood [1, 2] Research on relationships between various adipokines and endocrine neoplasms is ongoing.

Expression of adiponectin (AdipoR1 and AdipoR2) and leptin (Ob-R) receptors in adrenal tumors has not been widely investigated so far.

The authors of the current study were the first to retrospectively analyze the expression of adiponectin (AdipoR1 and AdipoR2) as well as leptin (Ob-R) receptors in adrenal tumor samples of patients who had been operated on in a single clinical center.

\section{Material and methods}

The study included 128 patients (87 females, 41 males) diagnosed with adrenal tumors who had been hospitalized in the Department of Endocrinology and Internal Medicine of the Medical University of Gdansk between 1995 and 2014. Patients were aged 23 to 92 (mean age: 52.5 years; females 53 years; males 51.3 years).

In the group of operated tumors, adrenal adenomas (CA) were diagnosed in 28 cases, cortical hyperplasia tumors $(\mathrm{CNH})$ in 35 cases, pheochromocytomas (PHEO) in 20 cases and malignant pheochromocytomas (PHEOM) in 5 cases.

All groups of patients were found to have comparable body mass index (BMI) (CC - BMI median: $26 \mathrm{~kg} / \mathrm{m}^{2}$; CNH/CA - BMI median: $27 \mathrm{~kg} / \mathrm{m}^{2}$; PHEO/PHEOM - BMI median: $26 \mathrm{~kg} / \mathrm{m}^{2}$ ).

The study material comprised tissue specimens of resected adrenal tumors. The histological type of the tumor was determined according to the
2004 World Health Organization Classification (Pathology and Genetics of Tumours of Endocrine Organs, Lyon, 2004).

Tumor tissue was preserved according to standard procedures (fixed for approximately $48 \mathrm{~h}$ in $10 \%$ formaldehyde solution, later dehydrated using appropriate alcohol and xylene solutions, and, finally, embedded in low melting point paraffin). Tissue specimens of analyzed cases were re-assessed in routinely prepared hematoxylin-eosin $(\mathrm{H}+\mathrm{E})$ stains to verify the histopathological diagnosis. From the studied specimens fragments most representative for neoplasm were chosen; there were neither necrotic lesions nor thermal damage areas in these fragments.

The selected specimens along with respective paraffin blocks were used to map tumor areas extracted for tissue microarrays. This was done using $1.5-\mathrm{mm}$ diameter needles. Tumor cores were inserted in pre-prepared "recipient" paraffin blocks that contained no tissue. The complete tissue microarrays were created using Manual tissue arrayer I by Beecher Instruments (MTAI, K7 BioSystems). At least three cores (biopsies) of neoplastic tissue were removed. This way at least ten microarrays were acquired. Additionally, in every newly created recipient block, cores of palatine tonsil and stomach wall were inserted, which served as an internal control and facilitated orientation among cores acquired from different specimens within one block. This orientation method allowed for localizing a specific core of a given tumor specimen.

The study was confirmed by the Independent Ethic Committee of the Medical University of Gdansk (NKBBN/360-98/2016).

\section{Preparation of specimens}

Immunohistochemical stains were made with microtome sections of 4 micrometer thickness. They were placed on Superfrost PLUS slides (by Surgipath) and later incubated overnight at $37^{\circ} \mathrm{C}$.

Antibodies used for detection along with applied methodology are presented in Table I.

\section{Assessment of immunohistochemical reactivity and statistical analysis}

Antigen expression was assessed in three samples acquired from the same tumor. The assess-

Table I. Antibodies used for detection along with applied methodology

\begin{tabular}{|lcccccc|}
\hline Target & $\begin{array}{c}\text { Catalog } \\
\text { number }\end{array}$ & Dilution & $\begin{array}{c}\text { Epitope } \\
\text { retrieval }\end{array}$ & $\begin{array}{c}\text { Incubation } \\
\text { time }\end{array}$ & Control tissue & $\begin{array}{c}\text { Method } \\
\text { of evaluation }\end{array}$ \\
\hline AdipoR1 & ab126611 & $1: 50$ & PTlink DAKO & $60^{\prime}$ & Endometrium & Semiquantitative \\
\hline AdipoR2 & LS-B9345 & $1: 50$ & PTlink DAKO & Overnight & Small intestine & Semiquantitative \\
\hline Ob-R & ab2139 & $1: 10$ & PTlink DAKO & $60^{\prime}$ & Placenta & Semiquantitative \\
\hline
\end{tabular}




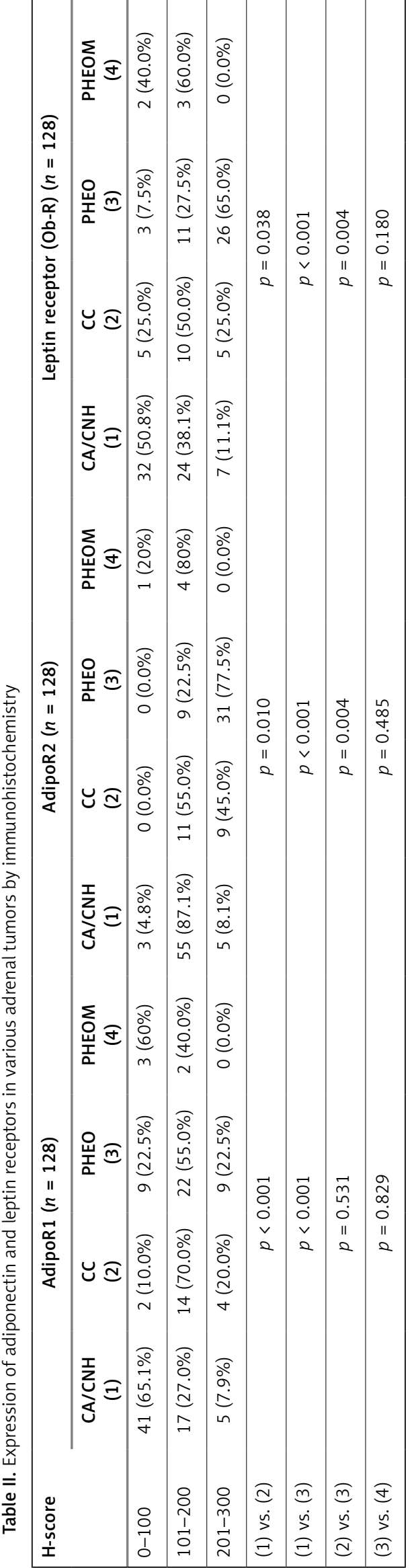

ment comprised the percentage of cells with positive reactivity $(0-100 \%)$ as well as the intensity of reactivity (0-3), and was expressed as their product (H-score). For each tumor three scores were calculated, and the highest of these was considered final.

$\mathrm{H}$-score values were classified as follows: 0 to 100 denoted lack of or low antigen expression, 101 to 200 denoted moderate antigen expression, and 201 to 300 denoted high antigen expression.

\section{Statistical analysis}

Standard descriptive statistics were calculated. Differences between groups were determined using linear regression with robust standard errors. Correlations were assessed using Spearman's method. $P$-values lower than 0.05 were considered statistically significant. Calculations were performed with STATA 13.1 software by StataCorp (USA, Texas).

To increase the reliability of the test results, two independent pathologists evaluated the results of immunohistochemistry in tissues of adrenal tumors. All ACC cases are defined according to the Weiss criteria.

\section{Results}

In two thirds of cortical adenoma and cortical nodular hyperplasia cases absence of or low AdipoR1 receptor expression was revealed. Its expression was significantly higher in cortical carcinomas $(p<0.001)$ and pheochromocytomas $(p<$ 0.001 ). There was no statistically significant difference between AdipoR1 expression in carcinomas and pheochromocytomas (Table II, Figure 1).

AdipoR2 was at least moderately expressed in all types of tumors. This receptor's expression was significantly higher in cortical carcinomas than in adenomas combined with nodular hyperplasia tumors $(p=0.010)$, as well as significantly higher in pheochromocytomas compared to cortical carcinomas $(p=0.004)$ (Table II, Figure 2).

Leptin receptor expression was absent or low in half of nodular hyperplasia tumors and adrenal cortex adenomas. Its expression was significantly higher in cortical cancers $(p=0.038)$. In pheochromocytomas this receptor was expressed more abundantly than in adrenocortical carcinomas $(p=0.004)$ (Table II, Figure 3).

Using retrospective data, the authors assessed the relationship between the expression of adrenal adipokine receptors and patient's sex and BMI.

There was no statistically significant difference between adrenal adipokine receptor expression levels and gender of the patients (AdipoR1 $p=$ 0.846; AdipoR2 $p=0.521$; Ob-R $p=0.642$ ). There was a significant difference between BMI and adipokine receptors' expression. Although higher BMI 

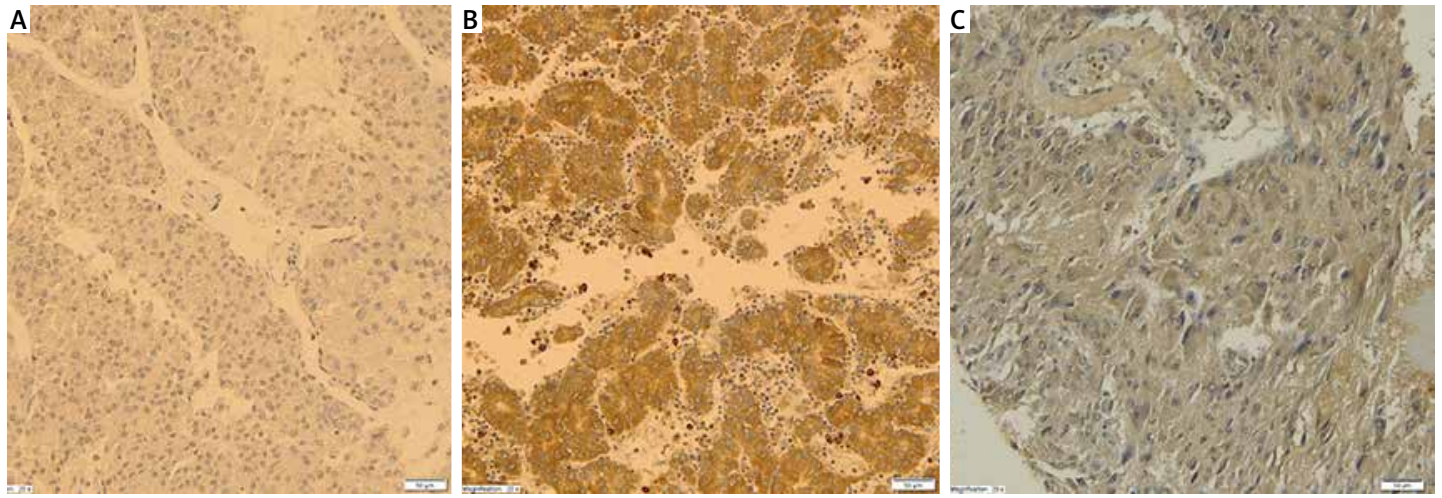

Figure 1. Expression of AdipoR1 in ACA (A), ACC (B), PHEO (C), magnification 20x
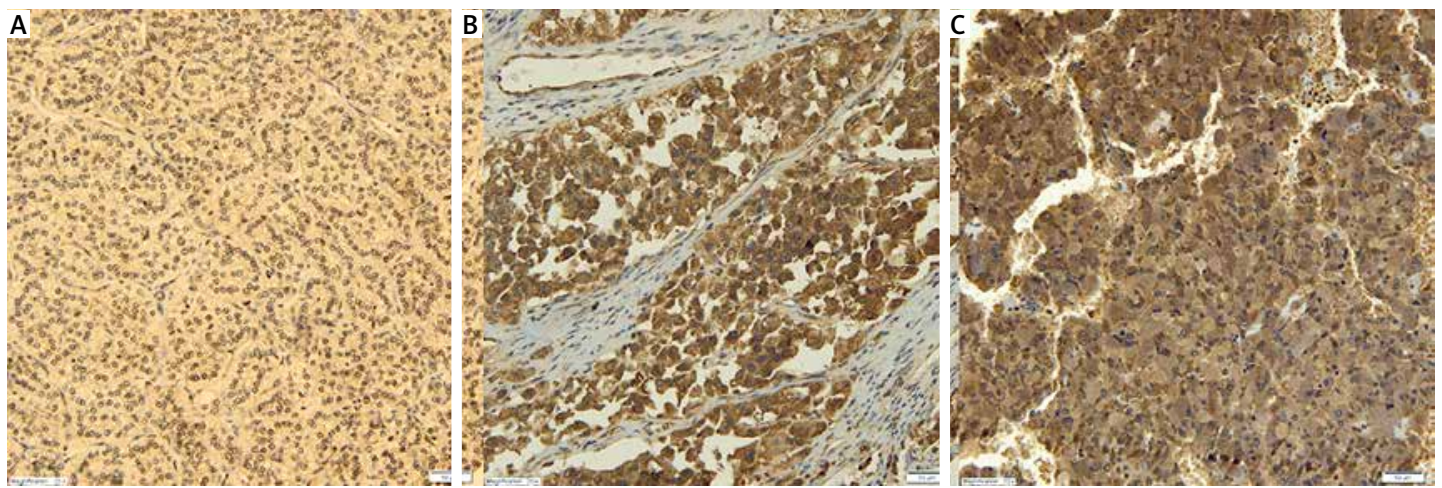

Figure 2. Expression of AdipoR2 in ACA (A), ACC (B), PHEO (C), magnification 20x
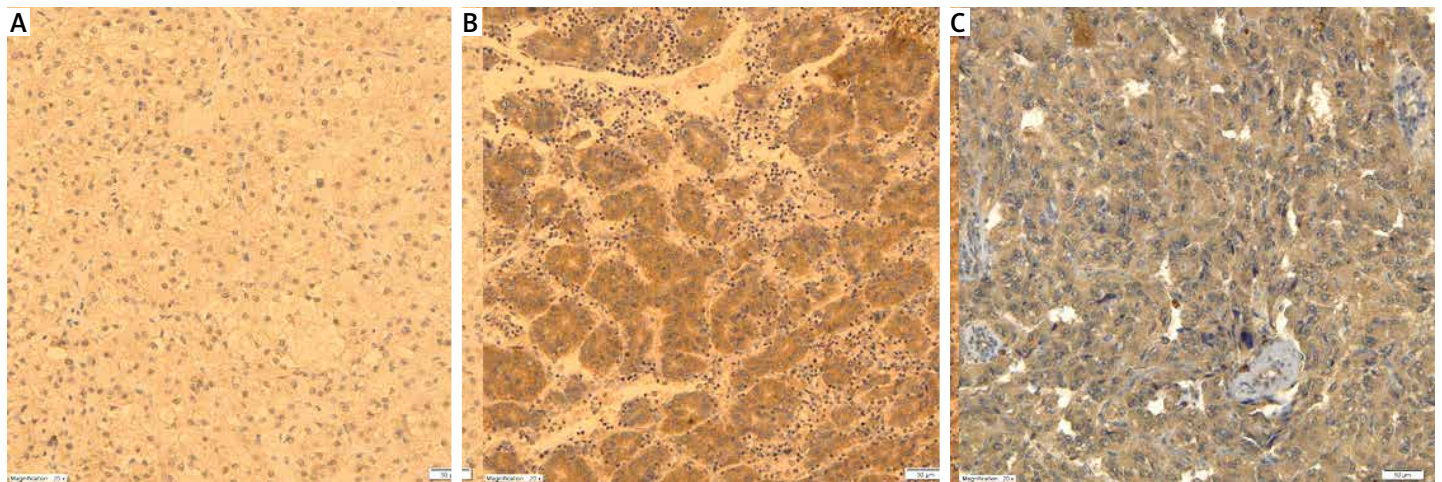

Figure 3. Expression of Ob-R in ACA (A), ACC (B), PHEO (C), magnification 20x

index was associated with lower adipokine receptors' expression (AdipoR1 $p=0.0337$; AdipoR2 $p=0.0001$; Ob-R $p=0.0008)$, the BMI index was similar in each histopathological group.

\section{Discussion}

The role of adipokines in the development of human endocrine neoplasms has not been elucidated. There have been few studies in which expression of adiponectin and leptin receptors in adrenal gland neoplasms in humans was investigated. However, the relationship between hormonal activity of adipose tissue and tumorigenesis as well as hormonal activity of adrenal tumors seems significant [1, 2].
The authors of one of the most recent studies showed that the adipose tissue surrounding adrenal glands actively secretes leptin and adiponectin [4]. Local adipokine secretion may have an effect on adrenal tumor pathophysiology [4].

ADIPOR 1 and ADIPOR2 genes are expressed physiologically in human adrenal glands. Two recently discovered adiponectin receptors, AdipoR1 and AdipoR2, mediate the biological effects of the adipose tissue-derived protein adiponectin [5].

Adiponectin receptors were expressed in many obesity-associated and -unassociated human neoplasms. Significantly higher AdipoR1 and AdipoR2 expression was found in patients with obesity-associated neoplasms compared to those neoplasms 
that probably are not etiologically associated with obesity, such as cervical squamous cell carcinoma, adrenocortical carcinoma [2] or thyroid cancer [3].

In our study, differential AdipoR1 and AdipoR2 expression in histopathologically distinct adrenal tumors from patients operated on in a single clinical center was examined for the first time. We found significantly higher AdipoR1 and AdipoR2 expression in adrenocortical cancer compared to benign neoplastic growth, i.e. cortical nodular hyperplasia and adenoma.

Several possible mechanisms may account for the role of adipokines in neoplasia. Elevated adiponectin plasma concentration improves insulin sensitivity in the obese. Therefore, in the case of low adiponectin levels elevated serum insulin and IGF 1 levels lead to increased proliferation, which also applies to neoplastic cells [1, 2, 6]. Adiponectin selectively binds with multiple mitogenic growth factors, which ultimately affects the regulation of proliferation, differentiation, and apoptosis through $5^{\prime}$-AMP-activated protein kinase (AMPK)/mammalian target of rapamycin (mTOR), and c-Jun NH2- terminal kinase and STAT3 (JNKSTAT3) pathways [7-9]. Adiponectin is also an angiogenesis inhibitor in endothelial cells $[2,7]$. In recent studies the role of AdipoR1 and AdipoR2 in neoplasms has been investigated. Ex vivo research showed that by binding with AdipoR1 adiponectin may attenuate the pro-neoplastic effect of leptin by its inhibitory action on the leptin receptor in the esophageal neoplasm $[8,10]$, or by inhibiting the progression to the $\mathrm{S}$ (synthesis) phase in breast cancer [11]. There are, however, no studies explaining the role of AdipoR2 in human neoplasms.

Increased adiponectin receptor expression in malignant adrenal tumor tissue may be explained by the down-regulation mechanism, which is present and has been elucidated in breast epithelial cancer [12] and Barrett's adenocarcinoma [13]. In the case of the current study, the authors deduced that low plasma adiponectin levels, associated with pro-neoplastic effects, lead to increased AdipoR1 and AdipoR2 expression in adrenal tumor tissue, with higher expression found in cortical cancers.

Isobe et al. were the first to reveal the presence of AdipoR1 and AdipoR2 in human pheochromocytoma (PHEO) tissue [14]. Pheochromocytoma are tumors that produce catecholamines, which results in reduced glucose tolerance. Diabetes is present in one third of patients with PHEO. The relationship between diabetes and pheochromocytoma encompasses suppression of insulin secretion by catecholamines and induction of insulin resistance [14].

So far, no studies have compared adiponectin receptors' expression in adrenal cortex and me- dulla neoplasms. We showed that AdipoR1 and AdipoR2 expression was significantly higher in pheochromocytomas compared to adrenocortical tumors. We found no statistically significant differences between the expression of adiponectin receptors in benign and malignant $\mathrm{PHEO}$. It should be remarked that there were only five cases of malignant pheochromocytomas in the studied group.

Adrenaline has an up-regulatory effect on adiponectin receptor expression. It seems that high catecholamine concentrations found in PHEO, which cause glucose intolerance and diabetes in $1 / 3$ cases, induce an intense compensatory increase in the expression of adiponectin receptors, AdipoR1 in particular, in PHEO tumor tissue, most likely due to a decrease in adiponectin plasma concentration in pheochromocytoma patients [14]. The authors of the current study speculate that catecholamine-mediated adiponectin suppression is more intense than the supposed effect of hormones secreted by adrenocortical tumors.

Leptin is one of the crucial factors that govern energy balance and body weight in humans. It is mainly synthesized by differentiated adipocytes, and it stimulates intracellular lipolysis of triglycerides of muscle cells, while it inhibits lipolysis in the liver and pancreatic islets [15]. Acting on its receptors located in the $\beta$-cells of pancreatic islets, and inhibiting the activation of ATP-dependent potassium channels, leptin reduces insulin synthesis. This hormone also has a role in the development of insulin resistance [15]. Expression of the leptin receptor Ob-R has been found in the adrenal glands of rats, mice and humans $[16,17]$.

Prior to the current study, a comparison of leptin receptor expression in histopathologically distinct adrenal cortex and medulla tumors has not been carried out. The authors of this report found no statistically significant differences in leptin receptor expression between benign and malignant pheochromocytomas, whereas leptin receptor expression was significantly higher in adrenocortical cancers compared to benign cortical neoplasms, i.e. nodular hyperplasia and adenoma of the adrenal cortex.

Mechanisms underlying the oncogenic effect of leptin have not been clarified thus far. It has been shown that the hormone stimulates the growth of neoplastic cells in esophageal, stomach, pancreatic, prostate, ovarian, and lung cancers [15]. The authors of another report suggested a relationship between leptin level and stimulation of proliferation of colon cancer cells [18]. It has also been indicated that leptin promotes proliferation of certain (not all) breast neoplasms in vitro, and promotes tumor invasiveness and angiogenesis in some animal models $[18,19]$. It seems that pro-neoplastic effects of leptin are more strongly pronounced in neoplasms associated with obesity 
(esophageal, breast, stomach, colon, and pancreatic cancers) compared to those in which obesity plays a lesser role [8].

An attempt at an assessment of the proliferative effect of leptin in adrenal tumors was undertaken by Glasgow and coworkers as early as 1999 [17]. They examined normal and cancerous tissue of the adrenal cortex. These researchers found that although the leptin receptor is present in adrenal tumors, leptin does not regulate the proliferation of neoplasms [17].

In our study a comparison between leptin receptor expression in adrenal cortical and medullary neoplasms was made. We found that this receptor's expression is significantly higher in PHEO than in adrenocortical tumors.

In a study by Bottner et al. it was shown that chronic supraphysiological synthesis of catecholamines present in PHEO patients does not lower the leptin level. It may be associated with tolerance of the adipose tissue to chronic catecholamine excess [20]. Mutual relationships between surrounding fat tissue and leptin secretion stimulation by catecholamines may be connected with increased expression of leptin $\mathrm{Ob}-\mathrm{R}$ receptor in PHEO.

The retrospective results of our research should be correlated with plasma adiponectin and leptin levels in patients with malignant or benign adrenal tumors in future prospective studies.

In conclusion, the authors of this study were the first to compare expression of adiponectin and leptin receptors' expression in histopathologically distinct adrenal tumors. Despite the fact that the expression of these receptors in adrenal tumors not associated with obesity is lower compared to neoplasms in which obesity is a vital etiological factor, the statistically significant differences in adiponectin receptors' expression between cortical cancers and benign neoplasms suggest that adiponectin has a role in tumorigenesis also in these neoplasms. The authors also demonstrated that leptin receptor expression differs between malignant and benign adrenal neoplasms. Leptin receptor expression was, however, significantly higher in pheochromocytoma than in cortical carcinoma. It is possible that leptin's impact on metabolic disorders is greater than on neoplasia. Higher adiponectin and leptin receptor expression in PHEO might result from a significantly greater effect of catecholamines on secretion of adipokines from adipose tissue than adrenal cortex hormones' effect on this secretion.

The obtained results were independent of BMI and gender of the study groups.

The pathophysiological roles of adiponectin and leptin and their receptors, as well as the potential predictive value of these hormones in assessing the risk of malignancy, recurrence, and treatment results, require further prospective research.

\section{Acknowledgments}

This research was supported with funds of the Medical University of Gdansk - grant number: ST-81.

\section{Conflict of interest}

The authors declare no conflict of interest.

\section{References}

1. Dalamaga M, Diakopoulos KN, Mantzoros CS. The role of adiponectin in cancer: a review of current evidence Endocr Reviews 2012; 33: 547-94.

2. Chou SH, Tseleni-Balafouta S, Moon HS, et al. Adiponectin receptor expression in human malignat tissues. Horm Canc 2010; 1: 136-45.

3. Mitsiades N, Pazaitou-Panayiotou K, Arionis KN, et al. Circulating adiponectin is inversely associated with risk of thyroid cancer: in vivo and in vitro studies. J Clin Endocrinol Metab 2011; 96: E2023-8.

4. Letizia C, Petramala L, Tiziana di Gioia CR, et al. Leptin and adiponectin mRNA expression from the adipose tissue surrounding the adrenal neoplasia. J Clin Endocrinol Metab 2015; 100: E101-4.

5. Rossi GP, Sticchi D, Giuliani L, et al. Adiponectin receptor expression in the human adrenal cortex and aldosterone-producing adenomas. Int J Mol Med 2006; 17: 975-80.

6. Ellulu MS, Patimah I, Khaza'ai H, Rahmat A, Abed Y. Obesity and inflammation: the linking mechanism and the complications. Arch Med Sci 2017; 13: 851-63.

7. Bråkenhielm E, Veitonmäki N, Cao R, et al. Adiponectin-induced antiangiogenesis and antitumor activity involve caspase-mediated endothelial cell apoptosis. Proc Nati Acad Sci USA 2004; 101: 2476-81.

8. Beales IL, Garcia-Morales C, Ogunwobi OO, Mutungi G. Adiponectin inhibits leptin-induced oncogenic signaling in oesophageal cancer cell by activation of PTP1B. Mol Cell Endocrinol 2014; 382: 150-8.

9. Wang Y, Lam KS, Xu JY, et al. Adiponectin inhibits cell proliferation by interacting with several growth factors in an oligomerization-dependent manner. J Biol Chem 2005; 280: 18341-7.

10. Ogunwobi OO, Beales IL. Globular adiponectin, activing via adiponectin receptor-1, inhibits leptin-stimulated oesophageal adenocarcinoma cell proliferation. Mol Cell Endocrinol 2008; 285: 43-50.

11. Nakayama S, Miyoshi Y, Ishihara H, Noguchi S. Growth-inhibitory effect of adiponectin via adiponectin receptor 1 on human breast cancer cell through inhibition of S-phase entry without inducing apoptosis. Breast Cancer Res Treat 2008; 112: 405-10.

12. Dos Santos E, Benaitreau D, Dieudonne MN, et al. Adiponectin mediates an antiproliferative response in human MDA-MB 231 breast cancer cell. Oncol Rep 2008; 20: 971-7.

13. Konturek PC, Burnat G, Rau T, Hahn EG, Konturek S. Effect of adiponectin and ghrelin on apoptosis of Barrett adenocarcinoma cell line. Dig Dis Sci 2008; 53: 253-6.

14. Isobe K, Fu L, Tatsuno I, et al. Adiponectin and adiponectin receptors in human pheochromocytoma. J Atheroscler Thromb 2009; 16: 442-7. 
15. Siemińska L. Adipose tissue. Pathophysiology, distribution, sex differences and the role in inflammation and cancerogenesis. Endocrinol Pol 2007; 58: 330-42.

16. Malendowicz LK, Rucinski M, Belloni AS, Ziolkowska A, Nussdorfer GG. Leptin and the regulation of the hypothalamic-pituitary-adrenal axis. Int Rev Cytol 2007; 263: 63-102.

17. Glasow A, Bornstein SR, Chrousos GP, Brown JW, Scherbaum WA. Detection of Ob-receptor in human adrenal neoplasms and effect of leptin on adrenal cell proliferation. Horm Metab Res 1999; 31: 247-51.

18. Husting SD, Berger NA. Energy balance, host-related factors, and cancer progression. J Clin Oncol 2010; 28: 4058-65.

19. Nkhata KJ, Ray A, Schuster TF, Grossmann ME, Cleary MP. Effects of adiponectin and leptin co-treatment on human breast cancer cell growth. Oncol Rep 2009; 21: 1611-9.

20. Bottner A, Eisenhofer G, Torpy DJ, et al. Lack of leptin suppression in response to hypersecretion of catecholamines in pheochromocytoma patients. Metabolism 1999; 48: 543-5. 